\title{
Content in YouTube Videos for Rosacea: Cross-sectional Study
}

Corey H Basch ${ }^{1}$, MPH, EdD; Priscila E Laforet ${ }^{2}$, MPH; Grace C Hillyer ${ }^{3}$, MPH, EdD; Erica J Seidel ${ }^{4}$, PsyD; Christie Jaime $^{1}$, MS

\author{
${ }^{1}$ Department of Public Health, William Paterson University, Wayne, NJ, United States \\ ${ }^{2}$ Division of Hematology/Oncology, Department of Medicine, Columbia University Irving Medical Center, New York, NY, United States \\ ${ }^{3}$ Department of Epidemiology, Mailman School of Public Health, Columbia University, New York, NY, United States \\ ${ }^{4}$ Department of Student Life/Counseling Center, Borough of Manhattan Community College, New York, NY, United States
}

\author{
Corresponding Author: \\ Corey H Basch, MPH, EdD \\ Department of Public Health \\ William Paterson University \\ 300 Pompton Rd \\ Wayne, NJ, 07470 \\ United States \\ Phone: 19737202603 \\ Email: baschc@wpunj.edu
}

\section{Abstract}

Background: Rosacea is an inflammatory skin disease that is chronic in nature. In addition to the physical symptoms, there are substantial quality of life issues that patients with rosacea experience, largely due to the visible nature in which rosacea manifests.

Objective: The purpose of this study was to describe the content related to rosacea in highly viewed English- and Spanish-language videos on YouTube.

Methods: We coded identifying information for each video and categories including characteristics of rosacea, clinical solutions, and alternative solutions. The 100 YouTube videos examined were viewed 18.5 million times between 2006 and 2020 , and 57.3\% $(10,652,665 / 18,592,742)$ of these views were of consumer videos.

Results: Videos posted by consumers more often promoted or were trying to sell a product or procedure (32/55, 58\% of consumers vs $10 / 31,32 \%$ of medical professionals and 4/14, $29 \%$ of television, internet, news, or entertainment sources; $P=.03$ ) and more frequently mentioned the use of makeup or other ways to cover up rosacea (30/55, 55\% of consumers vs $6 / 31,19 \%$ of medical professionals and $2 / 14,14 \%$ of television, internet, news, or entertainment sources; $P<.001)$. Videos sourced from medical professionals more often mentioned medication $(17 / 31,55 \%)$ than videos uploaded by consumers $(14 / 55,25 \%)$ or TV, internet, news, or entertainment sources $(3 / 14,21 \%)(P=.01)$.

Conclusions: Given that rosacea is experienced differently for each person, consumer advice that works for one individual may not work for another. There is a need for reliable videos on rosacea to emphasize this and clarify misconceptions.

(JMIR Dermatol 2021;4(1):e24517) doi: $\underline{10.2196 / 24517}$

\section{KEYWORDS}

rosacea; YouTube; social media; skin disease; skin; chronic; dermatology

\section{Introduction}

Rosacea is an inflammatory skin disease that is chronic in nature [1]. The cause of rosacea is unknown and the pathophysiology is inadequately comprehended [1]. Current statistics indicate that this is a prevalent problem, with an estimated 416 million adults affected [2] worldwide and an estimated 16 million individuals with rosacea in the United States [3]. Recent research has led to improved understanding of the common triggers and symptomology of this widely experienced issue. Triggers of rosacea include genetic and environmental factors [1], and patients are often encouraged to monitor environmental triggers closely to avoid the onset of symptoms.

The most commonly identifiable symptom of rosacea includes redness or flushing in the face, but the range of symptoms can be variable and are broken down into subtypes. The American Academy of Dermatology has identified 4 subtypes: (1) subtype 1 , which is characterized by visible redness, flushing, and blood 
vessels; (2) subtype 2, in which acne-like breakouts are common; (3) subtype 3, which is rare and involves thickening skin that can result in rhinophyma, a thickened and bulbous nose; and (4) subtype 4, which affects the eyes with issues ranging from burning and stinging to the development of cysts [4]. It is possible to experience more than one subtype at a time. Naturally, because subtypes are varied and may overlap, treatments are dependent upon related symptoms and patient experiences [5]. These treatments include but are not limited to topical therapies [6-9], antibiotics [10], laser and pulsed light therapies [11-15], and reconstructive surgery [16,17].

In addition to the physical symptoms, there are substantial quality of life issues that patients with rosacea experience, largely due to the visible nature in which rosacea manifests [4]. Much has been written and researched about the medical aspects of rosacea, such as causes, prognosis, and treatment, but the psychological impact of the condition is infrequently discussed and of great importance to those with this condition. With limited ability to control triggers and the lack of a cure, patients are challenged with a lifelong chronic condition that alters their facial appearance, which often impacts their self-esteem and quality of life, especially when the rosacea is severe [18-21].

Current research suggests that $90 \%$ of Americans use the internet [22], and many consumers search the web for information related to their health. YouTube is a highly popular medium for sharing information through videos, with an estimated 2 billion unique users [23]. Studies of YouTube are prevalent on a variety of health issues and issues concerning the skin specifically [24-27]. The purpose of this study was to describe the content related to rosacea in highly viewed English- and Spanish-language videos on YouTube.

\section{Methods}

The 100 videos with the most views on YouTube were identified using the keyword "rosacea" on May 31, 2020, and were recorded and coded. Videos in English and Spanish were included in the study. Six videos were not reviewed, as they were in a language other than English or Spanish, and they were replaced with the next 6 videos in English or Spanish.

Metadata were identified for each video, including the URL, source of video upload (consumer, medical professional, television- or internet-based news, or entertainment television), number of views, length of video in minutes and seconds, date of upload, language in which the video was recorded, and whether the video featured a medical doctor. A fact sheet from the American Academy of Dermatology was used to create coding categories for content [4]. Categories included characteristics of rosacea, clinical solutions, and alternative solutions.

Characteristics of rosacea included a general description of the condition, triggers and flare-ups of rosacea, the fact that rosacea is more common among women, and the impact of rosacea on the quality of life, such as feelings of frustration, embarrassment, worry, low self-esteem, anxiety, and depression. Mentions by subtype were noted. Specific details of the symptom subtype are noted below. Clinical solutions included mentions of surgery for thickened skin, laser treatment, medication, measures to protect against the sun, a potential cure, and the promotion of products or procedures. Skin care tips and mentions of makeup to cover the skin were included in the alternative solutions category. Responses were coded as "yes" or "no" for whether the video mentioned each of the above characteristics or solutions.

Frequencies and percentages of all categorical variables were calculated, and means, standard deviations, and ranges were determined for the number of views and video length. Video source was recoded as consumer, medical professional, or combined television-based news and entertainment television. Chi-square tests and analysis of variance were used to assess possible associations between video source and the characteristics and content of the videos. Authors EJS and CJ each coded half the videos and then coded a random $10 \%$ subset to ascertain interrater reliability. Using Cohen $\kappa(\kappa=0.92)$, interrater reliability was shown to be excellent. Because human subjects were not involved in this study, this protocol was not reviewed by an institutional review board, per the policies at William Paterson University and Columbia University.

\section{Results}

The 100 YouTube videos examined were viewed 18.5 million times between 2006 and 2020, and $57.3 \%$ $(10,652,665 / 18,592,742)$ of these views were of consumer videos (Table 1). 
Table 1. Characteristics of YouTube videos $(\mathrm{N}=100)$ about rosacea by video upload source.

\begin{tabular}{|c|c|c|c|c|c|}
\hline Characteristic & Total $(\mathrm{N}=100)$ & Consumer (n=55) & $\begin{array}{l}\text { Medical professional } \\
(\mathrm{n}=31)\end{array}$ & $\begin{array}{l}\text { Television- or internet- } \\
\text { based news and entertain- } \\
\text { ment television }(n=14)\end{array}$ & $P$ value \\
\hline \multicolumn{6}{|l|}{ Video characteristics } \\
\hline Views, n (\%) & $18,592,742(100)$ & $10,652,665(57.3)$ & $5,527,057(29.2)$ & $2,513,020(13.5)$ & $\mathrm{N} / \mathrm{A}^{\mathrm{a}}$ \\
\hline Views, mean (SD) & $\begin{array}{l}185,927 \\
(205,020)\end{array}$ & $\begin{array}{l}193,685 \\
(205,962)\end{array}$ & $175,066(210,611)$ & $179,501(202,362)$ & .45 \\
\hline Views, range & $33,076-1,407,672$ & $40,254-1,407,672$ & $42,443-1,003,575$ & $33,076-782,574$ & N/A \\
\hline Video length (min), mean (SD) & $10.53(8.92)$ & $11.45(8.60)$ & $9.20(7.53)$ & $9.93(12.60)$ & .42 \\
\hline Video length (min), range & $0.82-46.17$ & $0.83-46.17$ & $0.82-25.93$ & $1.60-43.32$ & N/A \\
\hline Video upload date, n (\%) & & & & & .15 \\
\hline 2006-2010 & $6(6.0)$ & $3(5.5)$ & $2(6.5)$ & $1(7.1)$ & \\
\hline 2011-2015 & $35(35.0)$ & $16(29.1)$ & $10(32.3)$ & $9(64.3)$ & \\
\hline $2016-2020$ & $59(59.0)$ & $26(47.3)$ & $19(61.3)$ & $4(28.6)$ & \\
\hline Language of video, $n(\%)$ & & & & & .03 \\
\hline English & $78(78.0)$ & $40(72.7)$ & $29(93.5)$ & $9(64.3)$ & \\
\hline Spanish & $22(22.0)$ & $15(27.3)$ & $2(6.5)$ & $5(35.7)$ & \\
\hline Features a medical professional, n (\%) & $33(33.0)$ & $1(1.8)$ & $27(87.1)$ & $5(35.7)$ & $<.001$ \\
\hline \multicolumn{6}{|l|}{ Characteristics of rosacea, $n(\%)$} \\
\hline Included general description of rosacea & $65(65.0)$ & $31(56.4)$ & $22(71.0)$ & $12(85.7)$ & .09 \\
\hline Mentions triggers and flare-ups & $52(52.0)$ & $23(41.8)$ & $19(61.3)$ & $10(71.4)$ & .07 \\
\hline $\begin{array}{l}\text { Mentions rosacea is more common in } \\
\text { women }\end{array}$ & $9(9.0)$ & $5(9.1)$ & $1(3.2)$ & $3(21.4)$ & .14 \\
\hline Mentions impact on quality of life & $30(30.0)$ & $19(34.5)$ & 7 (22.6) & $4(28.6)$ & .51 \\
\hline Mentions subtype 1 signs and symptoms ${ }^{\mathrm{b}}$ & $65(65.0)$ & $31(56.4)$ & $22(71.0)$ & $12(85.7)$ & .09 \\
\hline Mentions subtype 2 signs and symptoms ${ }^{c}$ & $65(65.0)$ & $30(54.5)$ & $23(74.2)$ & $12(85.7)$ & .04 \\
\hline Mentions subtype 3 signs and symptoms ${ }^{\mathrm{d}}$ & $33(33.0)$ & $12(21.8)$ & $14(45.2)$ & $7(50.0)$ & .03 \\
\hline Mentions subtype 4 signs and symptoms ${ }^{\mathrm{e}}$ & $14(14.0)$ & $6(10.9)$ & $3(9.7)$ & $5(35.7)$ & .04 \\
\hline \multicolumn{6}{|l|}{ Clinical solutions, $\mathbf{n}(\%)$} \\
\hline Mentions surgery for thickened skin & $3(3.0)$ & $1(1.8)$ & $0(0.0)$ & $2(14.3)$ & .03 \\
\hline Mentions laser treatment & $22(22.0)$ & $8(14.5)$ & $11(35.5)$ & $3(21.4)$ & .08 \\
\hline Mentions medication & $34(34.0)$ & $14(25.5)$ & $17(54.8)$ & $3(21.4)$ & .01 \\
\hline Mentions sun protection & $40(40.0)$ & $17(30.9)$ & $18(58.1)$ & $5(35.7)$ & .05 \\
\hline Mentions a cure & $8(8.0)$ & $5(9.1)$ & $1(3.2)$ & $2(14.3)$ & .41 \\
\hline Promotes or sells a product or procedure & $46(46.0)$ & $32(58.2)$ & $10(32.3)$ & $4(28.6)$ & .03 \\
\hline \multicolumn{6}{|l|}{ Alternative solutions, n (\%) } \\
\hline Promotes an alternative treatment & $31(31.0)$ & $17(30.9)$ & $8(25.8)$ & $6(42.9)$ & .52 \\
\hline Mentions skin care tips & $54(54.0)$ & $32(58.2)$ & $17(54.8)$ & $5(35.7)$ & .32 \\
\hline $\begin{array}{l}\text { Mentions makeup or other ways to cover } \\
\text { up rosacea }\end{array}$ & $38(38.0)$ & $30(54.5)$ & $6(19.4)$ & $2(14.3)$ & $<.001$ \\
\hline
\end{tabular}

${ }^{\mathrm{a}}$ N/A: not applicable.

${ }^{\mathrm{b}}$ Subtype 1 signs and symptoms: flushing and redness, particularly in the center of the face; visible broken blood vessels and spider veins; skin that is swollen, very sensitive, or may sting and burn; rough, dry, or scaling skin; and skin that tends to flush or blush easily.

${ }^{\mathrm{c}}$ Subtype 2 signs and symptoms: acne-like breakouts that tend to come and go and are found in the areas where the skin is very red, oily skin or skin that is very sensitive or may sting and burn, visible broken blood vessels and spider veins, and plaques with raised patches of skin. 
${ }^{\mathrm{d}}$ Subtype 3 signs and symptoms: bumpy skin or skin that begins to thicken, particularly on the nose, chin, forehead, cheeks, and ears; visible broken blood vessels and spider veins; oily skin; and large pores.

${ }^{\text {e }}$ Subtype 4 signs and symptoms: rosacea in the eyes where the eyes appear watery or bloodshot, feel gritty, burn or sting, itch, or are dry and sensitive to light; blurry or decreased vision; and visible broken blood vessels or a cyst on the eyelid.

The mean number of views was 185,927 (SD 205,020), and the mean length of the videos was 10.53 minutes (SD 8.92 minutes). Most videos were uploaded between 2016 and 2020 (59/100, $59.0 \%)$, recorded in English (78/100, 78.0\%), and did not feature a medical professional $(67 / 100,67.0 \%)$.

Consumer videos less often mentioned signs and symptoms of subtypes 2 and 3 (subtype 2: 30/55, 55\% of consumers vs 23/31, $74 \%$ of medical professionals and $12 / 14,86 \%$ of television or internet; $P=.04$; subtype $3: 12 / 55,22 \%$ of consumers vs $14 / 31$, $45 \%$ of medical professionals and $7 / 14,50 \%$ of television or internet; $P=.03$ ). Videos posted by consumers, however, more often promoted or were trying to sell a product or procedure $(32 / 55,58 \%$ of consumers vs 10/31, 32\% of medical professionals and 4/14, $29 \%$ of television, internet, news, or entertainment; $P=.03$ ) and more frequently mentioned the use of makeup or other ways to cover up rosacea $(30 / 55,55 \%$ of consumers vs $6 / 31,19 \%$ of medical professionals and $2 / 14$, $14 \%$ of television, internet, news, or entertainment; $P<.001$ ). Videos sourced from medical professionals more often mentioned medication $(17 / 31,55 \%)$ than videos uploaded by consumers $(14 / 55,25 \%)$ or television, internet, news, or entertainment sources $(3 / 14,21 \%)(P=.01)$. Videos uploaded from a television, internet, news, or entertainment source more often mentioned subtype $4(5 / 14,36 \%$ vs $6 / 55,11 \%$ of consumers and $3 / 31,10 \%$ of medical professionals; $P=.04)$ and surgical treatments for thickened skin $(2 / 14,14 \%)$ compared with consumer $(1 / 55,2 \%)$ and medical professional $(0 / 0,0 \%)$ videos $(P=.03)$.

\section{Discussion}

To our knowledge, this is the first study to examine the content of both English and Spanish rosacea videos on YouTube. The majority of the 100 most popular rosacea YouTube videos were uploaded by consumers. Thus, medical professionals should be aware that consumer opinions and thoughts on rosacea are accessed more often than professional materials. The type of information presented in the videos analyzed also varied depending on the source. Videos sourced from medical professionals were most likely to mention information on medication and the use of sun protection as treatments for rosacea, while videos sourced from consumers were most likely to mention information on alternative treatments like the use of makeup to cover up rosacea. Research indicates that cosmetics can exacerbate rosacea [28-30], and as such, the prevalent makeup tutorials related to covering rosacea could be promoting products that cause flares. In addition, rather than focusing on avoiding triggers, this content focused on hiding symptoms.
Within the context of the connection between self-esteem and body image [31], research is delving further into rosacea's social and emotional fallout. Patients may avoid social situations, retreat from relationships, or think negatively about themselves as a result of their symptoms. An increase in symptoms of depression and anxiety related to the severity of the rosacea have been reported [19]. Women are more likely to be diagnosed with rosacea, exacerbating the gaps in self-esteem that already exist between men and women [32], suggesting that, for women, a holistic approach to treating the condition may be warranted to affect both the psychological and physical manifestations of the disease [33].

Further, the videos sourced from consumers were also found to be the most likely to include information to sell a product. This discovery highlights that consumers may have various underlying motivations to upload videos on rosacea, such as commercial sponsorship, which might result in the communication of misinformation to increase sales of a sponsored product to treat rosacea. Analysis of the videos revealed that the accuracy and reliability of the information found in the videos varied greatly. This is best highlighted by the videos that included information on a cure despite the fact that there is no cure for rosacea. The findings of this study are similar to a prior study of rosacea on a variety of internet sources, including YouTube videos, which concluded that internet sources could contain peer-generated content that was harmful or misleading [34].

This study has limitations that warrant mention. The cross-sectional design indicates that data were only collected at one point in time, and given the fact that content on the internet is in flux, the most popular videos could change over time. Additionally, this study only included videos in English and Spanish despite videos being available in an array of languages. Further, there is no way to delineate who viewed each video and the reason they did so. Therefore, the study strictly offers insight on the content and coverage of information in the widely viewed videos on YouTube.

Nonetheless, this study offers insight into the content available on YouTube about rosacea. Given that rosacea is experienced differently for each person, consumer advice that works for one individual may not work for another. There is a need for reliable videos on rosacea to emphasize this and clarify misconceptions. Further study is needed on the accuracy and reliability of information on rosacea in videos sourced by consumers, as well as on the factors that influence consumers to create and upload videos on rosacea for YouTube.

\section{Conflicts of Interest}

None declared.

\section{References}


1. Buddenkotte J, Steinhoff M. Recent advances in understanding and managing rosacea. F1000Res 2018;7:1885 [FREE Full text] [doi: 10.12688/f1000research.16537.1] [Medline: $\underline{30631431]}$

2. Gether L, Overgaard L, Egeberg A, Thyssen J. Incidence and prevalence of rosacea: a systematic review and meta-analysis. Br J Dermatol 2018 Aug;179(2):282-289. [doi: 10.1111/bjd.16481] [Medline: 29478264]

3. National Rosacea Society. Rosacea now estmated to affect at least 16 million Americans. Rosacea Review. 2010. URL: https://www.rosacea.org/rosacea-review/2010/winter/rosacea-now-estimated-to-affect-at-least-16-million-americans [accessed 2020-09-15]

4. Rosacea: signs and symptoms. American Academy of Dermatology Association. 2020. URL: https://www.aad.org/public/ diseases/rosacea/what-is/symptoms [accessed 2020-09-21]

5. Johnson SM, Berg A, Barr C. Managing Rosacea in the Clinic: From Pathophysiology to Treatment-A Review of the Literature. J Clin Aesthet Dermatol 2020 Apr;13(Suppl 4):S17-S22 [FREE Full text] [Medline: $\underline{32802248]}$

6. Stein L, Kircik L, Fowler J, Tan J, Draelos Z, Fleischer A, et al. Efficacy and safety of ivermectin $1 \%$ cream in treatment of papulopustular rosacea: results of two randomized, double-blind, vehicle-controlled pivotal studies. J Drugs Dermatol 2014 Mar;13(3):316-323. [Medline: 24595578]

7. Taieb A, Khemis A, Ruzicka T, Barańska-Rybak W, Berth-Jones J, Schauber J, Ivermectin Phase III Study Group. Maintenance of remission following successful treatment of papulopustular rosacea with ivermectin $1 \%$ cream vs. metronidazole 0.75\% cream: 36-week extension of the ATTRACT randomized study. J Eur Acad Dermatol Venereol 2016 May;30(5):829-836. [doi: 10.1111/jdv.13537] [Medline: 26691278]

8. Fowler J, Jarratt M, Moore A, Meadows K, Pollack A, Steinhoff M, Brimonidine Phase II Study Group. Once-daily topical brimonidine tartrate gel $0.5 \%$ is a novel treatment for moderate to severe facial erythema of rosacea: results of two multicentre, randomized and vehicle-controlled studies. Br J Dermatol 2012 Mar;166(3):633-641 [FREE Full text] [doi:

10.1111/j.1365-2133.2011.10716.x] [Medline: 22050040]

9. Del Rosso JQ. Topical a-Agonist Therapy for Persistent Facial Erythema of Rosacea and the Addition of Oxmetazoline to the Treatment Armamentarium: Where Are We Now? J Clin Aesthet Dermatol 2017 Jul;10(7):28-32 [FREE Full text] [Medline: 29104721]

10. Del Rosso JQ, Leyden JJ, Thiboutot D, Webster GF. Antibiotic use in acne vulgaris and rosacea: clinical considerations and resistance issues of significance to dermatologists. Cutis 2008 Aug;82(2 Suppl 2):5-12. [Medline: 18924545]

11. Al-Niaimi F, Glagoleva E, Araviiskaia E. Pulsed dye laser followed by intradermal botulinum toxin type-A in the treatment of rosacea-associated erythema and flushing. Dermatol Ther 2020 Nov;33(6):e13976. [doi: 10.1111/dth.13976] [Medline: $\underline{32633449]}$

12. Papageorgiou P, Clayton W, Norwood S, Chopra S, Rustin M. Treatment of rosacea with intense pulsed light: significant improvement and long-lasting results. Br J Dermatol 2008 Sep;159(3):628-632. [doi: 10.1111/j.1365-2133.2008.08702.x] [Medline: 18565174$]$

13. Larson AA, Goldman MP. Recalcitrant rosacea successfully treated with multiplexed pulsed dye laser. J Drugs Dermatol 2007 Aug;6(8):843-845. [Medline: 17763618]

14. Tirico M, Jensen D, Green C, Ross E. Short pulse intense pulsed light versus pulsed dye laser for the treatment of facial redness. J Cosmet Laser Ther 2020 Mar 17;22(2):60-64. [doi: 10.1080/14764172.2020.1717540] [Medline: 32041440]

15. Kawana S, Ochiai H, Tachihara R. Objective evaluation of the effect of intense pulsed light on rosacea and solar lentigines by spectrophotometric analysis of skin color. Dermatol Surg 2007 Apr;33(4):449-454. [doi:

10.1111/j.1524-4725.2007.33092.x] [Medline: 17430379]

16. Saad M, Matteucci P. A very severe case of rhinophyma requiring a three-stage reconstruction with a forehead flap. Ann R Coll Surg Engl 2020 Oct;102(8):e219-e222. [doi: 10.1308/rcsann.2020.0164] [Medline: 32808811]

17. Kang JK, Lee JS, Choi JH, Shin MS, Yun BM. Surgical treatment of rhinophyma using an ordinary bur. Arch Craniofac Surg 2020 Aug;21(4):264-267 [FREE Full text] [doi: 10.7181/acfs.2020.00199] [Medline: $\underline{32867419}$ ]

18. Baldwin HE, Harper J, Baradaran S, Patel V. Erythema of Rosacea Affects Health-Related Quality of Life: Results of a Survey Conducted in Collaboration with the National Rosacea Society. Dermatol Ther (Heidelb) 2019 Dec;9(4):725-734 [FREE Full text] [doi: 10.1007/s13555-019-00322-5] [Medline: 31512178]

19. Egeberg A, Hansen PR, Gislason GH, Thyssen JP. Patients with Rosacea Have Increased Risk of Depression and Anxiety Disorders: A Danish Nationwide Cohort Study. Dermatology 2016;232(2):208-213. [doi: 10.1159/000444082] [Medline: 26954304]

20. Bewley A, Fowler J, Schöfer H, Kerrouche N, Rives V. Erythema of Rosacea Impairs Health-Related Quality of Life: Results of a Meta-analysis. Dermatol Ther (Heidelb) 2016 Jun;6(2):237-247 [FREE Full text] [doi: 10.1007/s13555-016-0106-9] [Medline: 26983751]

21. Bewley A, Fowler J, Schöfer H, Kerrouche N, Rives V. Erythema of Rosacea Impairs Health-Related Quality of Life: Results of a Meta-analysis. Dermatol Ther (Heidelb) 2016 Jun;6(2):237-247 [FREE Full text] [doi: 10.1007/s13555-016-0106-9] [Medline: 26983751]

22. Anderson M, Perrin A, Jiang J, Kumar M. 10\% of Americans don't use the internet. Who are they? Pew Research Center. 2019 Apr 22. URL: https://www.pewresearch.org/fact-tank/2019/04/22/some-americans-dont-use-the-internet-who-are-they/ [accessed 2020-09-16] 
23. YouTube for Press. YouTube. URL: https://www.youtube.com/about/press/ [accessed 2020-08-23]

24. Basch CH, Basch CE, Hillyer GC, Reeves R. YouTube Videos Related to Skin Cancer: A Missed Opportunity for Cancer Prevention and Control. JMIR Cancer 2015 Mar 02;1(1):e1 [FREE Full text] [doi: 10.2196/cancer.4204] [Medline: 28410167]

25. Basch CH, Brown AA, Fullwood MD, Clark A, Fung IC, Yin J. YouTube as a source of information on skin bleaching: a content analysis. Clin Exp Dermatol 2018 Jun;43(4):399-403. [doi: 10.1111/ced.13335] [Medline: 29265426]

26. Li W, Ma JE, Chan AA, Lee DJ, Smogorzewski J. Hidradenitis suppurativa through the lens of YouTube: a cross-sectional analysis [online ahead of print]. J Am Acad Dermatol 2020 Jun 06. [doi: 10.1016/j.jaad.2020.05.148] [Medline: 32522514]

27. St Claire KM, Rietcheck H, Patel R, Dunnick C, Dellavalle R. Dermatology on YouTube - an update and analysis of new trends. Dermatol Online J 2018 Dec 15;24(12):13030 [FREE Full text] [Medline: 30677792]

28. Mandal A. Rosacea and cosmetics (make-up). News Medical and Life Sciences. 2019. URL: https://www.news-medical.net/ health/Rosacea-and-Cosmetics-(Make-Up).aspx\#: :text=Rosacea\%20sufferers $\% 20$ tend $\% 20$ to\%20have, and $\% 20$ stinging\%20of\%20the\%20face [accessed 2020-09-21]

29. Jappe U, Schnuch A, Uter W. Rosacea and contact allergy to cosmetics and topical medicaments--retrospective analysis of multicentre surveillance data 1995-2002. Contact Dermatitis 2005 Feb;52(2):96-101. [doi: 10.1111/j.0105-1873.2005.00514.x] [Medline: $\underline{15725288]}$

30. Diepgen T, Weisshaar E. Contact dermatitis: epidemiology and frequent sensitizers to cosmetics. J Eur Acad Dermatol Venereol 2007 Sep;21(Suppl 2):9-13. [doi: 10.1111/j.1468-3083.2007.02381.x] [Medline: 17716286]

31. Clay D, Vignoles VL, Dittmar H. Body Image and Self-Esteem Among Adolescent Girls: Testing the Influence of Sociocultural Factors. J Res Adolescence 2005 Nov;15(4):451-477. [doi: 10.1111/j.1532-7795.2005.00107.x]

32. Stapleton P, Crighton GJ, Carter B, Pidgeon A. Self-esteem and body image in females: The mediating role of self-compassion and appearance contingent self-worth. Humanistic Psychol 2017 Sep;45(3):238-257. [doi: 10.1037/hum0000059]

33. Huynh TT. Burden of Disease: The Psychosocial Impact of Rosacea on a Patient's Quality of Life. Am Health Drug Benefits 2013 Jul;6(6):348-354 [FREE Full text] [Medline: 24991368]

34. Riddoch LH. It takes one to know one: exploring patient dialogue on rosacea web-based platforms and their potential for significant harm. J Dermatolog Treat 2019 Feb;30(1):52-62. [doi: 10.1080/09546634.2018.1468067] [Medline: 29676186]

Edited by G Eysenbach; submitted 22.09.20; peer-reviewed by P Kumar, P Pan; comments to author 30.10.20; revised version received 31.10.20; accepted 15.01.21; published 10.02.21

Please cite as:

Basch CH, Laforet PE, Hillyer GC, Seidel EJ, Jaime C

Content in YouTube Videos for Rosacea: Cross-sectional Study

JMIR Dermatol 2021;4(1):e24517

URL: http://derma.jmir.org/2021/1/e24517/

doi: $10.2196 / 24517$

PMID:

CCorey H Basch, Priscila E Laforet, Grace C Hillyer, Erica J Seidel, Christie Jaime. Originally published in JMIR Dermatology (http://derma.jmir.org), 10.02.2021. This is an open-access article distributed under the terms of the Creative Commons Attribution License (https://creativecommons.org/licenses/by/4.0/), which permits unrestricted use, distribution, and reproduction in any medium, provided the original work, first published in JMIR Dermatology Research, is properly cited. The complete bibliographic information, a link to the original publication on http://derma.jmir.org, as well as this copyright and license information must be included. 